\title{
Hybrid Diffusion Imaging in Mild Traumatic Brain Injury
}

\author{
Yu-Chien Wu, Sourajit M. Mustafi, Jaroslaw Harezlak, ${ }^{2}$ Chandana Kodiweera, \\ Laura A. Flashman, and Thomas W. McAllister ${ }^{5}$
}

\begin{abstract}
Mild traumatic brain injury (mTBI) is an important public health problem. Although conventional medical imaging techniques can detect moderate-to-severe injuries, they are relatively insensitive to mTBI. In this study, we used hybrid diffusion imaging (HYDI) to detect white matter alterations in 19 patients with mTBI and 23 other trauma control patients. Within 15 days (standard deviation=10) of brain injury, all subjects underwent magnetic resonance HYDI and were assessed with a battery of neuropsychological tests of sustained attention, memory, and executive function. Tract-based spatial statistics (TBSS) was used for voxel-wise statistical analyses within the white matter skeleton to study betweengroup differences in diffusion metrics, within-group correlations between diffusion metrics and clinical outcomes, and between-group interaction effects. The advanced diffusion imaging techniques, including neurite orientation dispersion and density imaging (NODDI) and q-space analyses, appeared to be more sensitive then classic diffusion tensor imaging. Only NODDI-derived intra-axonal volume fraction $\left(\mathrm{V}_{\mathrm{ic}}\right)$ demonstrated significant group differences (i.e., 5-9\% lower in the injured brain). Within the mTBI group, $\mathrm{V}_{\text {ic }}$ and a q-space measure, $\mathrm{P}_{0}$, correlated with 6 of 10 neuropsychological tests, including measures of attention, memory, and executive function. In addition, the direction of correlations differed significantly between groups $\left(R^{2}>0.71\right.$ and $\left.p_{\text {interation }}<0.03\right)$. Specifically, in the control group, higher $\mathrm{V}_{\text {ic }}$ and $\mathrm{P}_{0}$ were associated with better performances on clinical assessments, whereas in the mTBI group, higher $\mathrm{V}_{\text {ic }}$ and $\mathrm{P}_{0}$ were associated with worse performances with correlation coefficients $>0.83$. In summary, the NODDI-derived axonal density index and q-space measure for tissue restriction demonstrated superior sensitivity to white matter changes shortly after mTBI. These techniques hold promise as a neuroimaging biomarker for mTBI.
\end{abstract}

Keywords: axonal density; clinical outcomes; hybrid diffusion imaging; mild traumatic brain injury; NODDI; q-space imaging

\section{Introduction}

$\mathbf{T}$ RaUmatic BRAIN INJURY (TBI) is an important public health problem, with approximately 1.6 million injuries occurring each year in the United States, the majority $(\sim 80 \%)$ of which are categorized as "mild.", 1,2 Individuals with mild traumatic brain injury (mTBI) typically experience symptoms such as headache, difficulty thinking, memory problems, attention deficits, mood swings, and frustration shortly after injury. These symptoms can persist in $10-20 \%$ of those with $\mathrm{mTBI}^{3,4}$ Further, people with mTBI may be at higher risk for neurodegenerative diseases, ${ }^{5,6} \mathrm{fu}-$ ture $\mathrm{TBI}^{7}$ and, in cases of repetitive injuries, chronic traumatic encephalopathy. ${ }^{8,9}$ Interestingly, despite these sequelae, findings from conventional magnetic resonance imaging (MRI) and computed tomography exams are often normal. To date, diagnosis of mTBI is made on clinical grounds, often based on self-report of clinical symptoms, which is subjective. As yet, there is no neuroimaging biomarker of sufficient sensitivity and specificity to be considered a valid and reliable indicator of mTBI. ${ }^{10,11}$

Diffuse axonal injury is generally believed to be the initial neuropathology associated with mTBI. ${ }^{12-18}$ The inertia forces subsequent to linear and rotational acceleration caused by TBIs may initiate cellular and subcellular biochemistry reactions, ${ }^{12}$ such as calcium dysregulation, ${ }^{13}$ thereby compromising cell membrane permeability ${ }^{14,15}$ and leading to morphological changes in axonal microstructures. In a study of a mouse model of closed TBI, injured

${ }^{1}$ Department of Radiology and Imaging Sciences, Indiana University School of Medicine, Indianapolis, Indiana.

${ }^{2}$ Department of Epidemiology and Biostatistics, School of Public Health, Indiana University, Bloomington, Indiana.

${ }^{3}$ Dartmouth Brain Imaging Center, Dartmouth College, Hanover, New Hampshire.

${ }^{4}$ Department of Psychiatry, Geisel School of Medicine at Dartmouth and Dartmouth-Hitchcock Medical Center, Lebanon, New Hampshire.

${ }^{5}$ Department of Psychiatry, Indiana University School of Medicine, Indianapolis, Indiana.

(c) Yu-Chien Wu et al., 2018; Published by Mary Ann Liebert, Inc. This Open Access article is distributed under the terms of the Creative Commons Attribution Noncommercial License (http://creativecommons.org/licenses/by-nc/4.0/) which permits any noncommercial use, distribution, and reproduction in any medium, provided the original author(s) and the source are credited. 
axons underwent dramatic pathomorphological changes with initial swelling and beading, followed by degeneration and apoptosis. ${ }^{16}$ Also shown in a fluid-percussion mouse model, such axonal injury progresses over a period of 4-6 weeks and significantly correlates with cognitive impairments. ${ }^{17}$ In a recent study of a mouse closedhead mTBI model, mice lacking a key mediator of Wallerian degeneration, the Sarml gene, exhibit attenuated axonal injury with better functional outcomes. ${ }^{18}$

Despite evidence of axonal injury from animal models, traumatic axonal injury has been more difficult to detect in human mTBI with neuroimaging. MRI-based methods, particularly diffusion tensor imaging (DTI), may be more sensitive than conventional imaging for detecting axonal injury. ${ }^{19-21}$ Among DTI metrics, fractional anisotropy (FA) is the most studied in TBI and is often used as an indicator of white matter "integrity." Although abnormal FA has been reported in patients with $\mathrm{mTBI}$, its direction of change and brain regions involved have varied, ${ }^{21-23}$ suggesting that further studies of diffusion-weighted techniques are needed. For example, several studies have reported reduced FA values in $\mathrm{mTBI},{ }^{24-31}$ whereas others have reported increased FA values. ${ }^{32-38}$ Even when controlling for the population source, injury-to-imaging interval, age of injury, sample size, publication date, acquisition parameters, and analysis methods, bidirectional changes in FA are reported in the literature. ${ }^{21,39}$ The lack of consensus across studies suggests fundamental limitations of DTI for detecting specific damage in mTBI.

Mathematically, FA is defined as the square root of the variance of the diffusion tensor eigenvalues divided by the square root of the sum of the squares of the eigenvalues. That means, in the model of cylindrical symmetry, that FA is driven by two forces-axial diffusivity (AD; principal eigenvalue) and radial diffusivity (RD; second and third eigenvalues that are perpendicular to the axis of the fiber). Therefore, differences in FA could be caused by changes in $\mathrm{AD}, \mathrm{RD}$, or both, making FA sensitive to many types of brain pathology $\mathrm{y}^{40,41}$ as well as susceptible to imaging noise, ${ }^{42,43}$ loss of specificity, and a lack of information about the nature of the underlying pathology. In addition, there are limitations in the assumptions underlying DTI. Although DTI successfully describes water diffusion behavior at modest bvalues (e.g., $1000 \mathrm{~s} / \mathrm{mm}^{2}$ ), many studies have demonstrated that simple Gaussian diffusion models do not sufficiently describe water diffusion in complex tissues, such as crossing fibers ${ }^{40,44-48}$ and mixtures of diffusion compartments. ${ }^{4-52}$ Thus, although diffusion-weighted imaging should theoretically be a significant advance in the detection and explication of the mechanisms of injury in mTBI, conventional DTI approaches have not yet reached this potential.

A novel diffusion MRI acquisition approach, hybrid diffusion imaging (HYDI), ${ }^{53}$ comprises multiple diffusion-weighting shells to distinguish diffusion compartments with different diffusivities and multiple diffusion-weighting directions in each shell to capture the directionalities of the compartments. HYDI data can be fit into the recently developed diffusion model, neurite orientation dispersion and density imaging (NODDI), to extract diffusion metrics that may be more biologically specific and unbiased by crossing fibers. ${ }^{54-57}$ Under the NODDI framework, whereas the fiber fanning/crossing is explained by an orientation dispersion index, the volume fraction of the intra-axonal compartment describes axonal density. ${ }^{54}$ In recent studies, the axonal density index computed by the NODDI model is almost identical to values obtained from electron microscopy (EM) of ex vivo mouse brains and is in good agreement with the previously reported fiber density of the human brain obtained using EM. ${ }^{58}$ In a study of human post-mortem spinal cord with multiple sclerosis (MS), the NODDI axonal density index demonstrated significant sensitivity to the histologically derived density of neuronal element (i.e., neurofilaments staining fraction). ${ }^{59}$ NODDI has been demonstrated to be more sensitive than classic DTI in long-term effects of sports-related concussion. ${ }^{60}$ Therefore, for mTBI characterized by diffuse axonal injury, the NODDI-derived metrics are highly relevant and may be valuable neuroimaging biomarkers.

Alternatively, changes of white matter microscopic morphology may be studied by a nonparametric analysis, q-space imaging. The q-space approach estimates the water diffusion function, the probability density function, also called mean apparent propagator $^{61}$ or ensemble average propagator. ${ }^{62,63}$ The zero displacement probability, $\mathrm{P}_{0}$, is the return to origin probability and presents the probability of those water molecules having no net diffusion within a diffusion time. $\mathrm{P}_{0}$ is often interpreted as a measure of restricted diffusion and cellularity. ${ }^{53,61,64}$ In animal studies of dysmyelination, $\mathrm{P}_{0}$ was found to be highly sensitive to myelination and brain maturation, ${ }^{65,66}$ consistent with other studies of $\mathrm{P}_{0}$ in demyelination of the human brain with MS. ${ }^{67,68}$

In this study, we used a five-shell HYDI to sample full q-space diffusion signals. The HYDI data are versatile and were analyzed using DTI, multi-compartmental NODDI modeling, and a nonparametric q-space approach. ${ }^{55,65,69,70}$ Seven diffusion metrics computed from the HYDI data were studied voxelwise within a common white matter skeleton with tract-based spatial statistics (TBSS). Diffusion metrics were compared between cohorts and correlated with cognitive function tests.

\section{Methods \\ Participants}

A total of 42 patients were recruited from the emergency department of the Dartmouth-Hitchcock Medical Center (Lebanon, NH), a level 1 trauma center. The mTBI group included 19 patients (11 females, 8 males) with recent autopedestrian accidents. Cases of moderate-to-severe TBI with positive clinical imaging findings (e.g., significant impact-site lesions and hemorrhage) were excluded from this study. In addition, an "other injury" control group consisting of 23 trauma control patients (11 females, 12 males) was also studied. Inclusion criteria for the mTBI group followed the guidelines of the American Congress of Rehabilitation Medicine, ${ }^{71}$ including an alteration in level of consciousness subsequent to a force acting on the head, a Glasgow Coma Score of 13-15, loss of consciousness $<30 \mathrm{~min}$, and post-traumatic amnesia $<24 \mathrm{~h}$. For the trauma control group, individuals with orthopedic injuries, but without injury involving the head, were screened for recruitment. Participants were excluded if they had a history of previous TBI, a history of other neurological disorders, significant systemic medical illness, a current substance-use disorder, and current Diagnostic and Statistical Manual of Mental Disorders, 4th Edition (DSM-IV) Axis I psychiatric illness based on Structured Clinical Interview for DSM interview. ${ }^{72}$ All participants provided informed consent approved by the guidelines of the institutional review board at Geisel School of Medicine at Dartmouth College (Lebanon, NH).

\section{Neuropsychological assessment}

Clinical assessments included a full battery of neuropsychological measures similar to our previous publications. ${ }^{10,73,74}$ The battery assessed the level of estimated intellectual function (Wide Range Achievement Test, 4th edition; WRAT4), sustained 


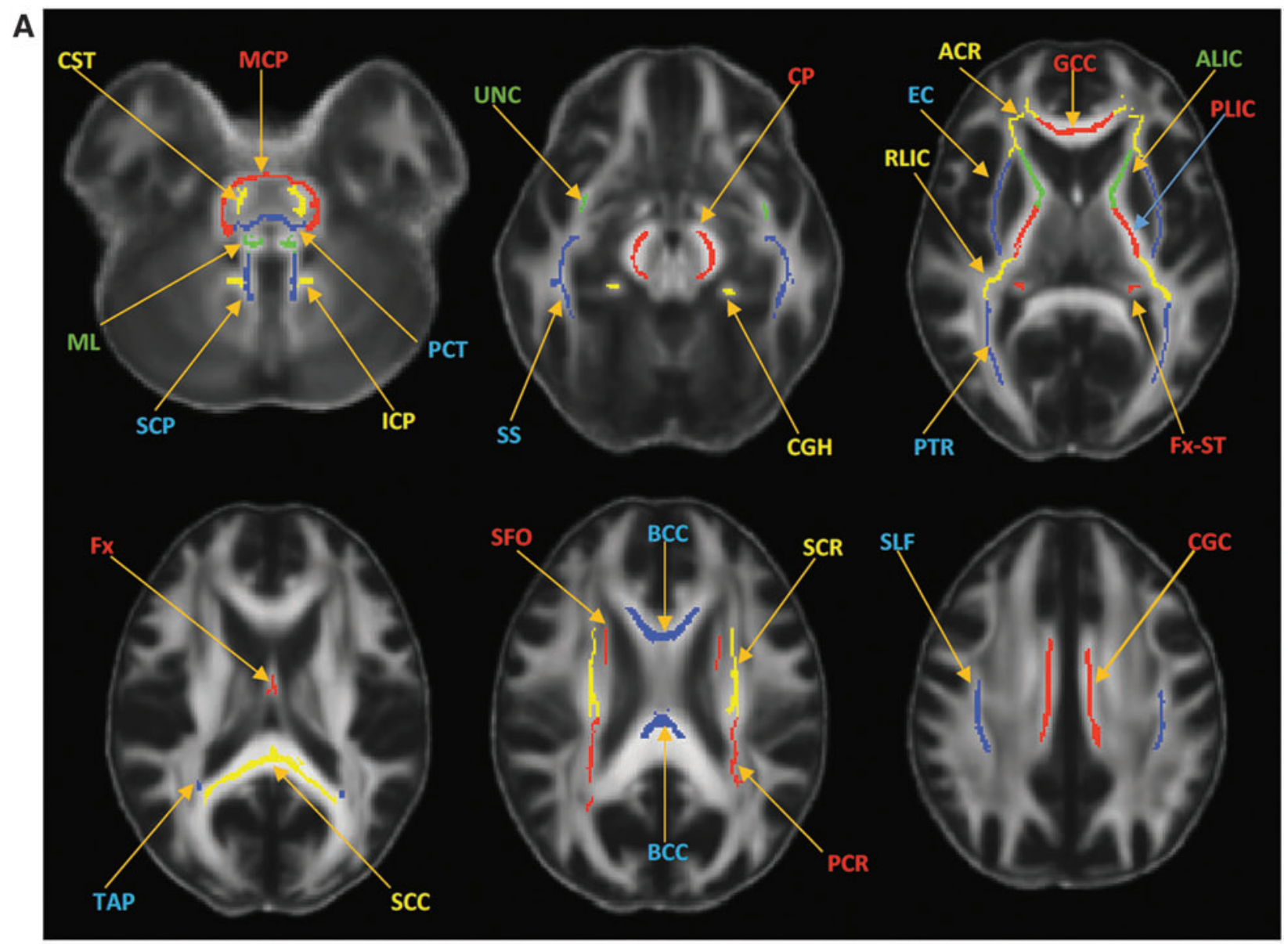

\begin{tabular}{|c|l|c|l|}
\hline ROI & & ROI & \\
\hline & Anterior Corona radiata & PCR & Posterior corona radiata \\
\hline ALIC & Anterior Limb of internal Capsule & PCT & Pontine Crossing tract \\
\hline BCC & Body of Corpus callosum & PLIC & Posterior limb of internal capsule \\
\hline CGC & Cingulum (cingulate gyrus) & PTR & Posterior thalamic radiation \\
\hline & Cingulum (hippocampus) & & Retro-lenticular part of IC \\
\hline CP & Cerebral peduncle & & Splenium of corpus callosum \\
\hline & Corticospinal tract & SCP & Superior cerebellar peduncle \\
\hline EC & External Capsule & & Superior corona radiata \\
\hline Fx & Fornix (column and body of fornix) & SFO & Superior fronto-occipital fasciculus \\
\hline Fx-ST & Fornix/Stria terminals & SLF & Superior longitudinal fasciculus \\
\hline GCC & Genu of corpus callosum & SS & Sagittal stratum \\
\hline & Inferior cerebellar peduncle & TAP & Tapetum \\
\hline MCP & Middle cerebellar Peduncle & UNC & Uncinate fasciculus \\
\hline ML & Medial lemniscus & & \\
\hline
\end{tabular}

FIG. 1. Skeletonized Johns Hopkins University (JHU) white matter atlas and their acronyms. (A) Skeletonized JHU white matter atlas are overlaid on the mean fractional anisotropy (FA) map of the 42 subjects in the standard MNI space. Skeletonization was performed by intersecting the original JHU atlas provided in FSL with the study-specific white matter skeleton of the 42 subjects. (B) A list of acronyms for the $48 \mathrm{JHU}$ atlas with matched colors in (A). MNI, Montreal Neurological Institute; ROI, region of interest. 


\section{Image processing}

Image processing included an initial pre-processing and diffusion metrics computation. The diffusion-weighted images were first denoised using a local principle component analysis (LPCA) approach provided in the toolbox developed by Manjon and colleagues. ${ }^{79}$ With the $b_{0}$ images as reference, the diffusion-weighted images were then corrected for motion, eddy current artifacts, and geometrical distortion using the $e d d y$ command provided in the FMRIB Software Library (FSL). ${ }^{80}$ Using an in-house MATLAB script (The MathWorks, Inc., Natick, MA), the transformation matrices output from the eddy command were used to rotate the corresponding diffusion-weighting directions to match the rotation of the brain image during the motion correction process. After image pre-processing, seven diffusion metrics were computed from the HYDI data set. Diffusion metrics of DTI were computed similarly to a previous work ${ }^{81}$ from the inner two shells of the HYDI data using the FSL dtifit command and included FA, AD, RD, and mean diffusivity (MD). NODDI metrics included the axonal density metric (intra-axonal volume fraction; $V_{i c}$ ) and orientation dispersion (OD) index, which were computed according to the three-compartment model described in previous works ${ }^{54,82}$ using a MATLAB toolbox provided in https://www.nitrc.org/projects/ noddi_toolbox/. The HYDI q-space analysis used in-house MATLAB scripts (The MathWorks, Inc.) ${ }^{69}$ to produce a diffusion metric, zero displacement probability $\left(\mathrm{P}_{0}\right)$, which describes restricted diffusion. ${ }^{53,64}$ The restricted diffusion index, $\mathrm{P}_{0}$, may infer cellularity of the tissue ${ }^{61,83}$ Maps of the diffusion metrics were nonlinearly transformed to standard Montreal Neurological Institute (MNI) space using Advanced Neuroimaging Tools (ANTs) registration. ${ }^{84}$

\section{Tract-based spatial statistics analysis}

To minimize partial volume effects in finite imaging resolution, in the standard MNI space, a common whole-brain while matter skeleton was extracted using the FSL toolbox, TBSS. ${ }^{85}$ The white matter skeleton included only voxels in the center of white matter tracts and excluded edge voxels, which may be contaminated with signals from the nearby anatomy. Within the white matter skeleton, nonparametric permutation-based statistics utilized in TBSS (i.e., the randomise command) was used for voxel-wise statistical analyses in which general linear models controlling for participants' sex, age, and intellectual ability (i.e., WRAT4) were tested. A threshold-free cluster enhancement $^{86}$ and 5000 permutations to obtain a corrected $p$ value ${ }^{87}$ were utilized in this study. White matter voxels were deemed significant as a corrected $p$ value $<0.05$ after being adjusted for multiple comparisons by controlling family-wise error rate. Using TBSS, we tested: 1) between-group differences in the diffusion metrics; 2) within-group correlations between the diffusion metrics and neuropsychological measures to explore how sensitive the diffusion metrics are to each neuropsychological outcome; and 3)

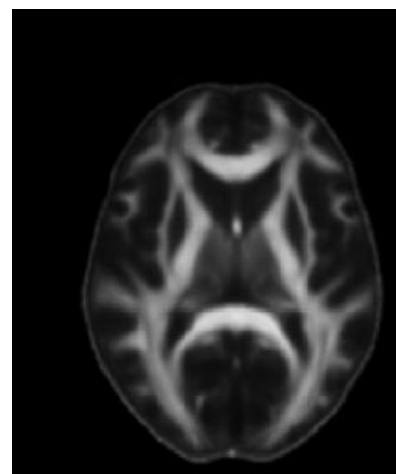

FA

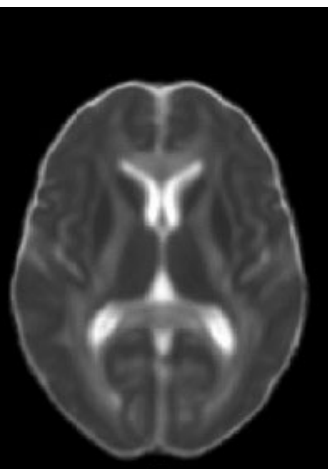

AD

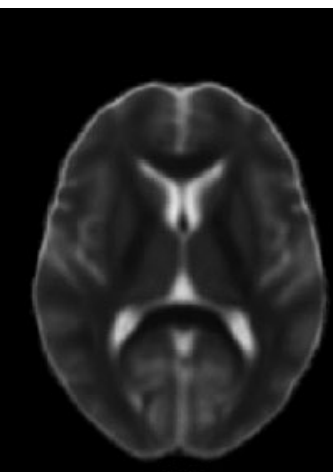

RD
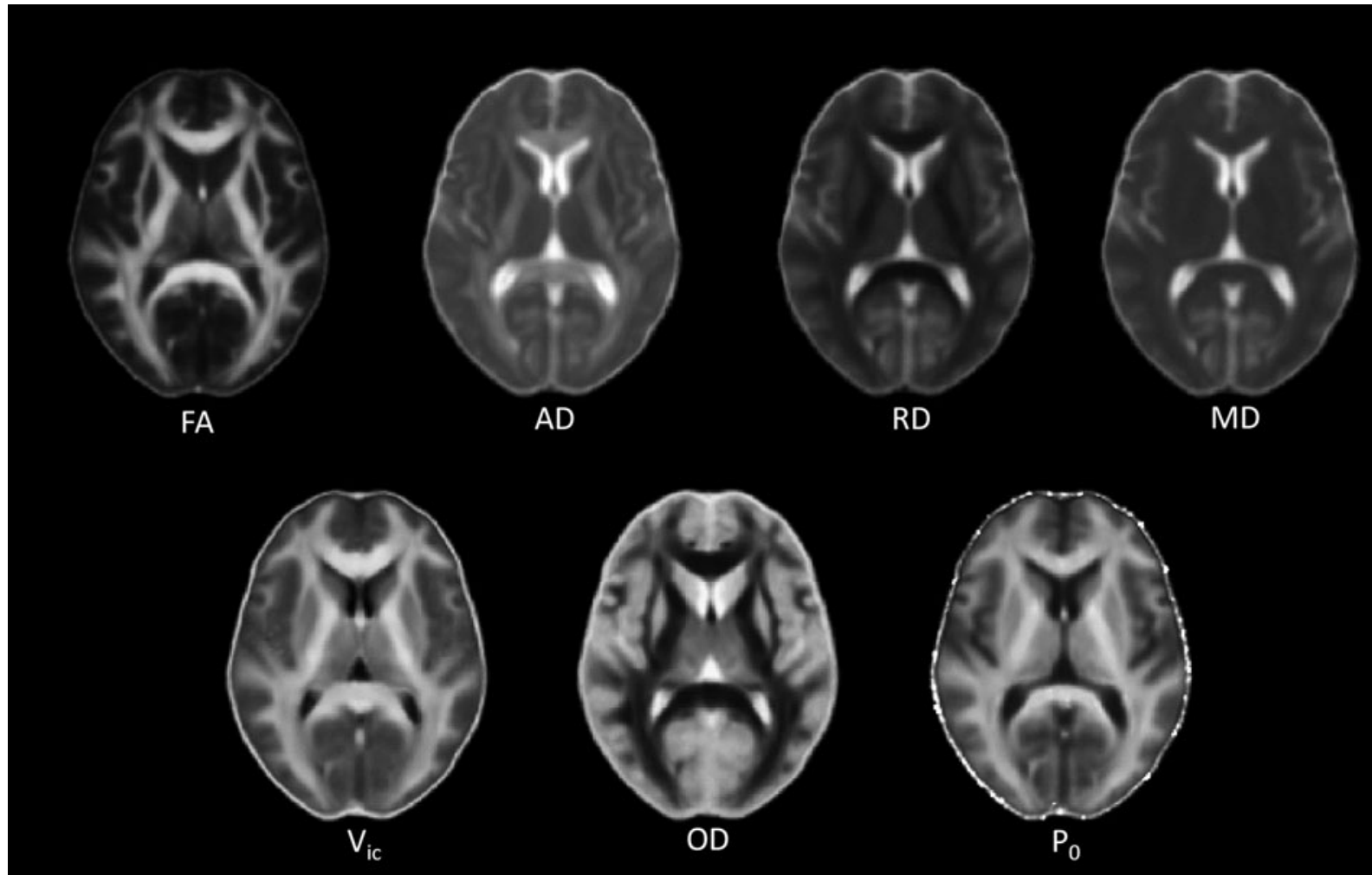

OD

FIG. 2. Maps of averaged diffusion metrics of the 42 subjects in the MNI standard space. Diffusion tensor imaging (DTI) metrics include fractional anisotropy (FA), axial diffusivity (AD), radial diffusivity (RD), and mean diffusivity (MD). Neurite orientation dispersion and density imaging (NODDI) metrics include axonal density $\left(\mathrm{V}_{\mathrm{ic}}\right)$ and orientation dispersion (OD). The q-space imaging metric is the zero displacement probability $\left(\mathrm{P}_{0}\right)$ describing the tissue restriction. The gray scale is 0 to $3 * 10^{-6} \mathrm{~mm}^{2} / \mathrm{s}$ for the diffusivities and is 0 to 1 for FA, $\mathrm{V}_{\mathrm{ic}}$, OD, and $\mathrm{P}_{0}$. High intensity in the FA and OD maps indicates high tissue coherence and low dispersion and vice versa. AD and RD have high white matter contrast only in compact fiber tracts with known single-fiber bundles, such as the corpus callosum and the internal capsule. The $\mathrm{P}_{0}$ map shows higher intensity in more restricted areas (i.e., white matter), as expected. White matter also has a higher intensity than gray matter in the $\mathrm{V}_{\mathrm{ic}}$ map, indicating higher axonal density. MNI, Montreal Neurological Institute. 

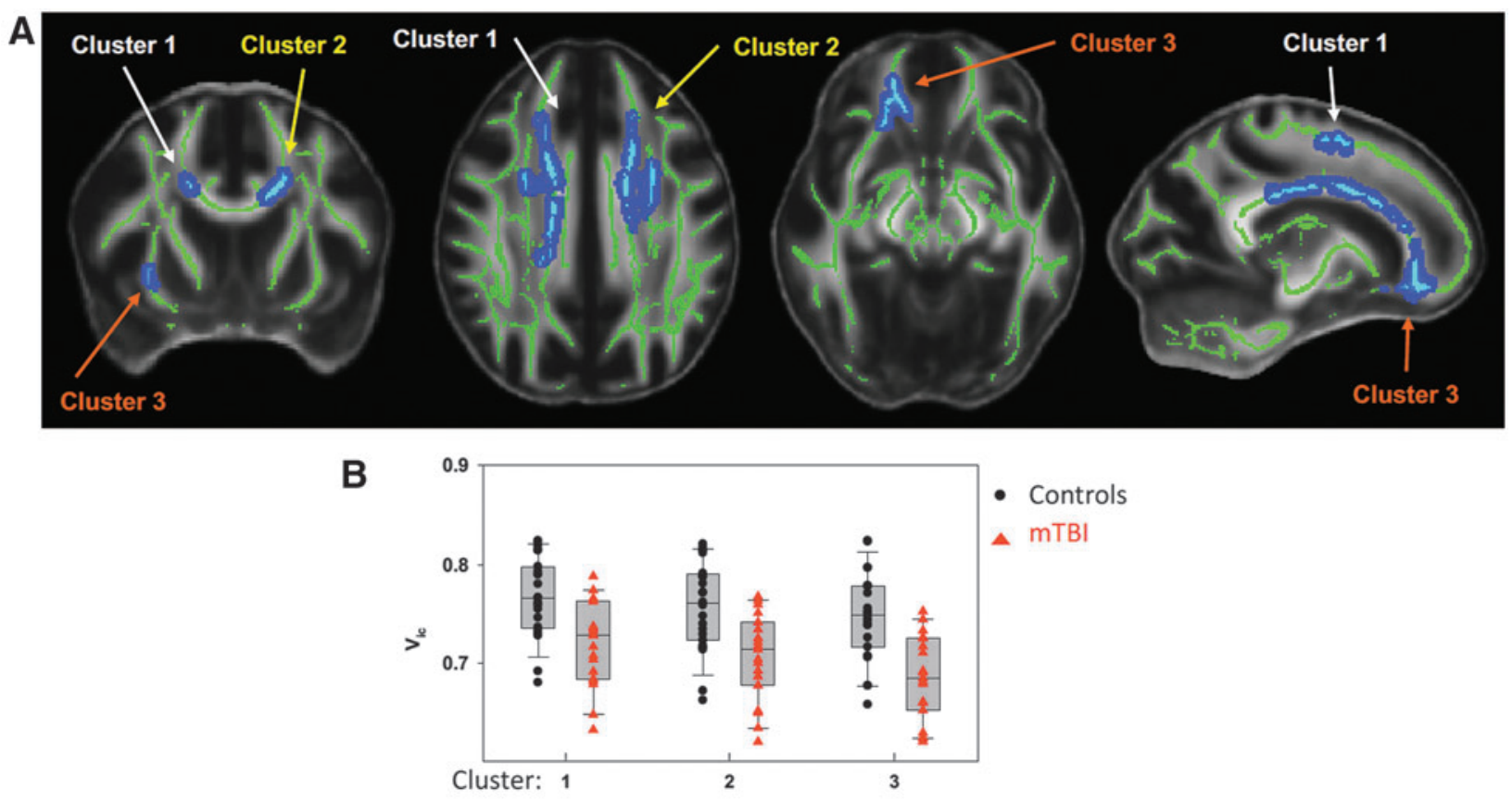

\begin{tabular}{|c|c|c|c|}
\hline Cluster & Size (voxel) & $\min \boldsymbol{P}$ & Anatomical regions \\
\hline 1 & 1266 & 0.045 & Body of Corpus Callosum, Anterior and Superior Corona radiata (Right) \\
\hline 2 & 863 & 0.042 & Body of Corpus Callosum, Anterior and Superior Corona radiata (left) \\
\hline 3 & 860 & 0.041 & Anterior Corona radiata (right), Genu of Corpus callosum \\
\hline
\end{tabular}

FIG. 3. Results of between-group differences in axonal density, $V_{i c}$. (A) Significant maps of tract-based spatial statistics (TBSS) using a general linear model. Green voxels denote white matter skeleton where the statistical test was performed. Light blue denotes voxels having significant differences in $\mathrm{V}_{\mathrm{ic}}$ between groups at $p<0.05$ adjusted for multiple comparisons using family-wise error rate (FWER). The dark blue is background enhancement for illustration purposes. Three separated significant clusters can be appreciated on the maps. (B) Results of the post-hoc analyses on the means of $V_{\text {ic }}$ in the three clusters using two-tailed Student's $t$-test with Statistical Package for the Social Sciences (SPSS, Inc., Chicago, IL). Black dots denote the mean $V_{i c}$ for each subject in the trauma control group, and red triangles denote the mean $\mathrm{V}_{\text {ic }}$ for each subject in the mTBI group. Consistently across the three clusters, the mTBI group demonstrated significantly decreased axonal density at $p<0.05$. (C) The table lists the size, minimum $p$, and anatomical distribution of the three clusters. Anatomical labeling was performed by intersecting the clusters with the Johns Hopkins University (JHU) 48 skeletonized white matter atlas shown in Figure 1. mTBI, mild traumatic brain injury.

how mTBI moderates the correlations, that is, the interaction of the group membership and the relationship between the neuropsychological measures and diffusion metrics in general linear regression analyses.

\section{Post-hoc region-of-interest analysis}

To produce aggregate results at the subject level, post-hoc region-of-interest (ROI) analyses were performed. For each subject, the mean of each diffusion metric was computed in an ROI comprising white matter voxels that were tested significant in TBSS. For between-group differences, a box plot was used with subjects' means plotted according to their group membership. For within-group correlations, a scatter plot was used with subjects' means plotted against their neuropsychological outcome measures. Further, post-hoc regression analyses allow us to retrieve correlation coefficients $(\rho)$ at the ROI level. In addition, to compare across the diffusion metrics, the total numbers of voxels that were significant in the TBSS regression analysis of within-group correlations and group interactions were summarized in a matrix format for each diffusion-neuropsychological pair. The anatomical interpretation of ROI was based on the Johns Hopkins University ( JHU) white matter atlas provided in
FSL $^{88}$ after skeletonization (Fig. 1). In particular, the TBSSsignificant ROIs were intersected with the JHU 48 white matter labels and the voxels counts were summarized in a color-coded matrix.

\section{Statistical analysis}

Outliers in the neuropsychological measures were detected and excluded if outside the median \pm 1.5 interquartile range (IQR). For between-group comparisons, neuropsychological outcome measures were analyzed with a two-tailed Student's $t$-test using the Statistical Package for the Social Sciences (version 24; SPSS, Inc., Chicago, IL). Between-group differences in diffusion metrics, within-group correlation, and interaction effects of mTBI were analyzed by regression analyses of general linear models controlling for participants' sex, age, and estimated intellectual ability (i.e., WRAT4).

\section{Results}

\section{Clinical and neuropsychological assessments}

Clinical reading of the conventional T1-weighted, T2weighted, and FLAIR images were normal and without 


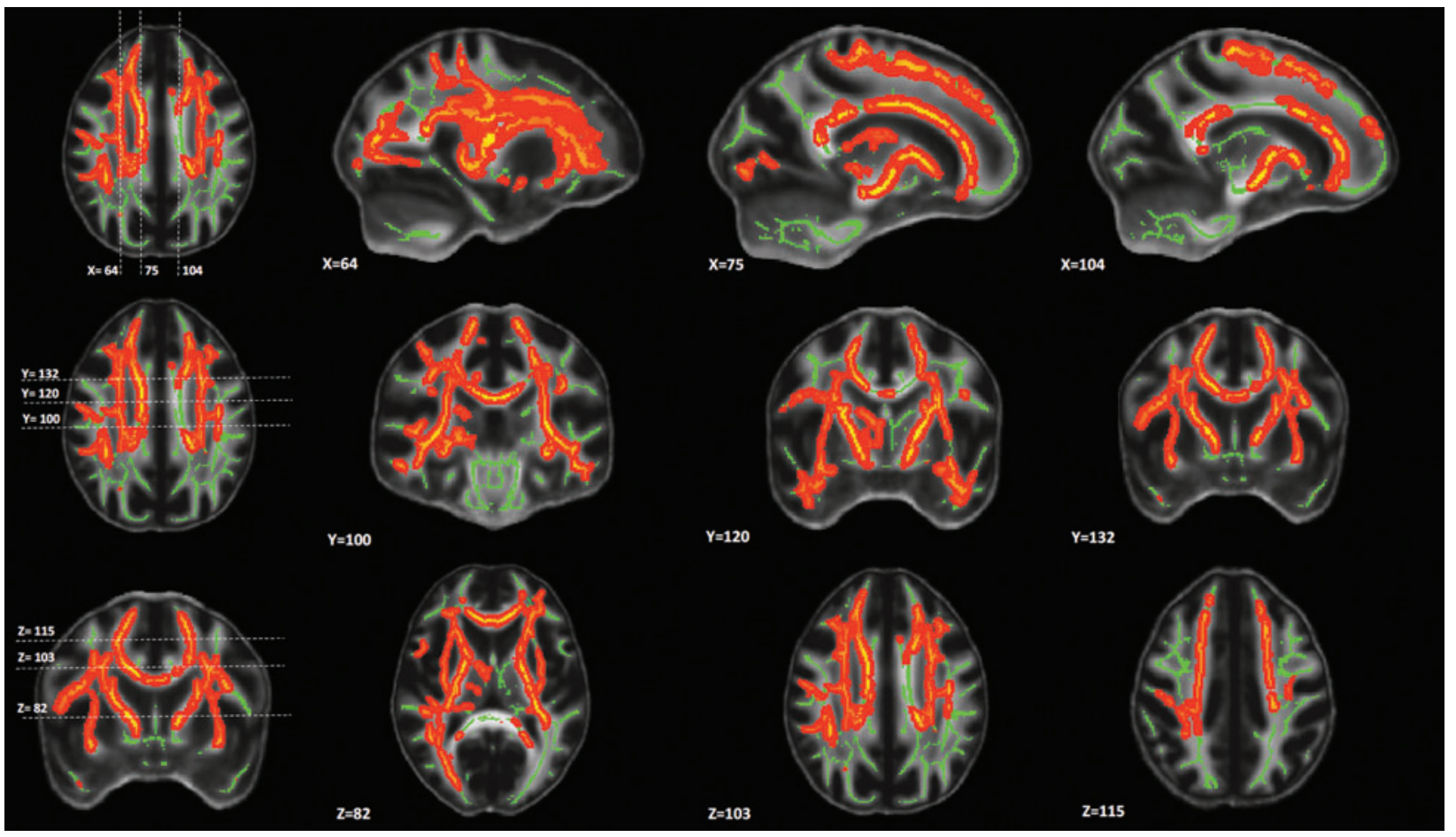

FIG. 4. TBSS maps of significant correlations between axonal density, $V_{i c}$, and Number Letter Switching from the DKEFS Trail Making Test (DKEFS4). In the mild TBI group, a wide spread of white-matter voxels had significant correlations. The mid-brain and posterior part of the brain, however, seemed to be spared. The total number (i.e., $\sim 5^{*} 10^{4}$ ) of significant voxels in this map is reported in Figure $3 \mathrm{~A}$ for the $\mathrm{V}_{\text {ic }}$-DKEFS4 pair. The scatter plot of each subject's DKEFS4 score and the averaged $\mathrm{V}_{\text {ic }}$ over the significant voxels are shown in Figure 3B (bottom middle panel). In addition, anatomical distributions of significant voxels in this map are reported in Figure 3C (row DKEFS4). TBSS, tract-based spatial statistics.

hemorrhage or incidental findings in all but 9 participants. Four mTBI patients and 5 trauma controls had nonspecific hyperintense foci in T2-weighted and FLAIR images with otherwise normal white matter. Further, there were no positive findings in CT examinations for all participants. Age distribution and length of education were similar in both groups (Table 1). Individuals with mTBI had a slightly higher verbal intellectual score (WRAT4) than trauma controls; thus, WRAT4 scores were included as a covariate in related statistical tests. None of the neuropsychological and mood measure results differed between groups, except for the distractibility condition of the CPT and RPQ. Distractibility reaction time (DISRT) and severity of PCSs (RPQ-3, RPQ13, and RPQ-TOT) were significantly higher (worse) in individuals with mTBI (Table 1). Although the injury-to-imaging interval had a relatively large standard deviation ( $\mathrm{sd} ; \mathrm{sd}=10$ days vs. a mean of 15 days; Table 1), there were no correlations between diffusion metrics and injury-to-imaging interval in a separated analysis using a general linear model.

\section{Between-group differences in the diffusion metrics}

Averaged maps of $\mathrm{FA}, \mathrm{AD}, \mathrm{RD}, \mathrm{MD}, \mathrm{V}_{\mathrm{ic}}, \mathrm{OD}$, and $\mathrm{P}_{0}$ across all subjects are shown in Figure 2. Among the seven diffusion metrics tested by TBSS, only $\mathrm{V}_{\text {ic }}$ differed significantly between groups (Fig. 3A). As shown in the bar plots of the post-hoc ROI analyses (Fig. 3B), individuals with mTBI exhibited lower $\mathrm{V}_{\text {ic. }}$. The amount of difference was approximately $5-9 \%$ of the $\mathrm{V}_{\text {ic }}$ mean value in trauma controls. These significant results (corrected $p<0.05$ ) were detected in three major clusters of white matter voxels with a minimum $p$ of 0.041 and a size $\geq 860 \mathrm{~mm}^{3}$ (Fig. 3C). Affected white matter tracts were located predominantly in the corpus callosum and its projections, including the anterior and superior corona radiata.

FIG. 5. Results of the within-group correlations for participants with mild TBI. (A) The color-coded matrix summarizes the total number of white matter voxels having significant correlations between the seven diffusion metrics (horizontal) and 10 neuropsychological measures (vertical) in the TBSS analyses. An example TBSS analysis of $\mathrm{V}_{\mathrm{ic}}$-DKEFS4 is shown in Figure 2. The cold colors indicate negative correlations, whereas the warm colors indicate positive correlations. (B) Results of post-hoc regression analyses between $\mathrm{V}_{\text {ic }}$ and the six neuropsychological measures that had significant TBSS results in (A). Each red triangle denotes 1 individual's neuropsychological score and mean $V_{\mathrm{ic}}$ over the significant voxels. The correlation coefficient and significance of the regression lines are reported in $\rho$ and $p$. (C) Anatomical distributions of significant white matter voxels in the TBSS analyses of correlations between $\mathrm{V}_{\text {ic }}$ and the 10 neuropsychological measures. The horizontal axis denotes the Johns Hopkins University (JHU) white matter labels in Figure 1 with the length of the interval reflecting its three-dimensional size (in cubic root) in the atlas. The color denotes the percentage (\%) of voxels in the labeled white-mater tract with significant correlations. TBI, traumatic brain injury; TBSS, tract-based spatial statistics. 
A

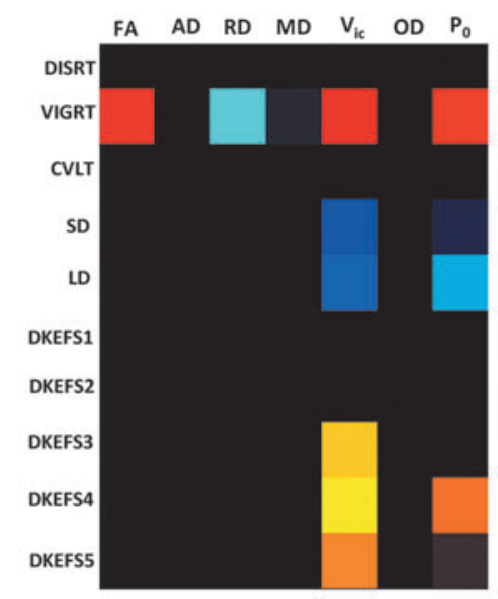

Negative correlation

Positive correlation:

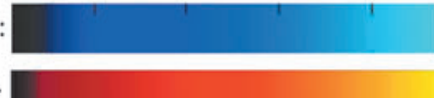

B
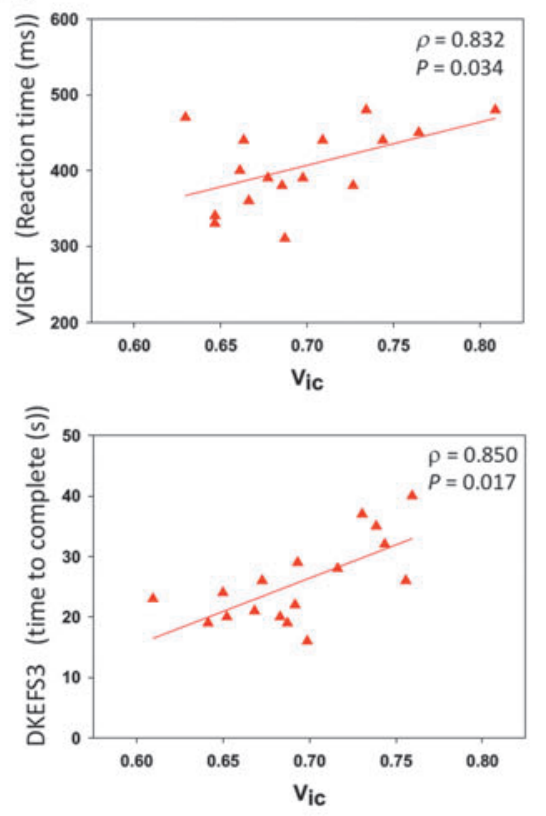
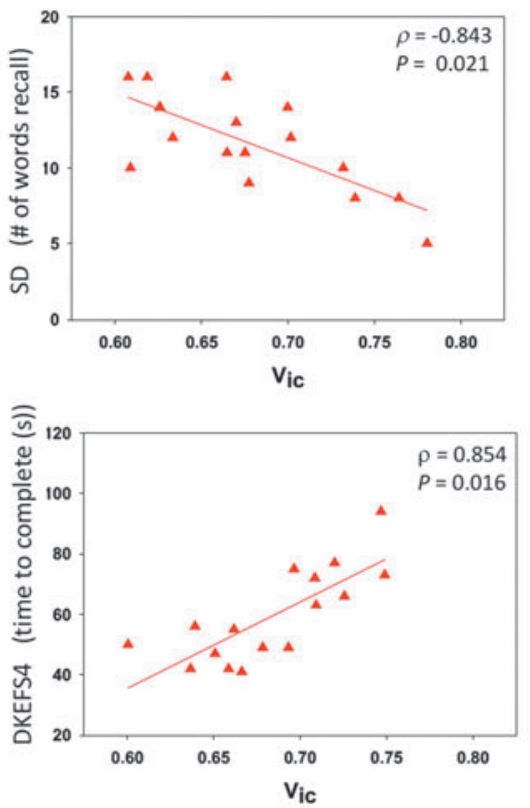
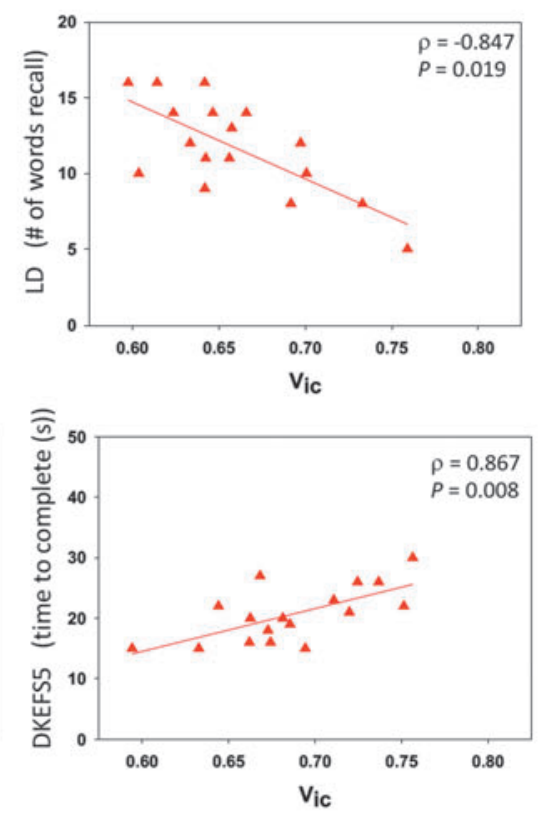

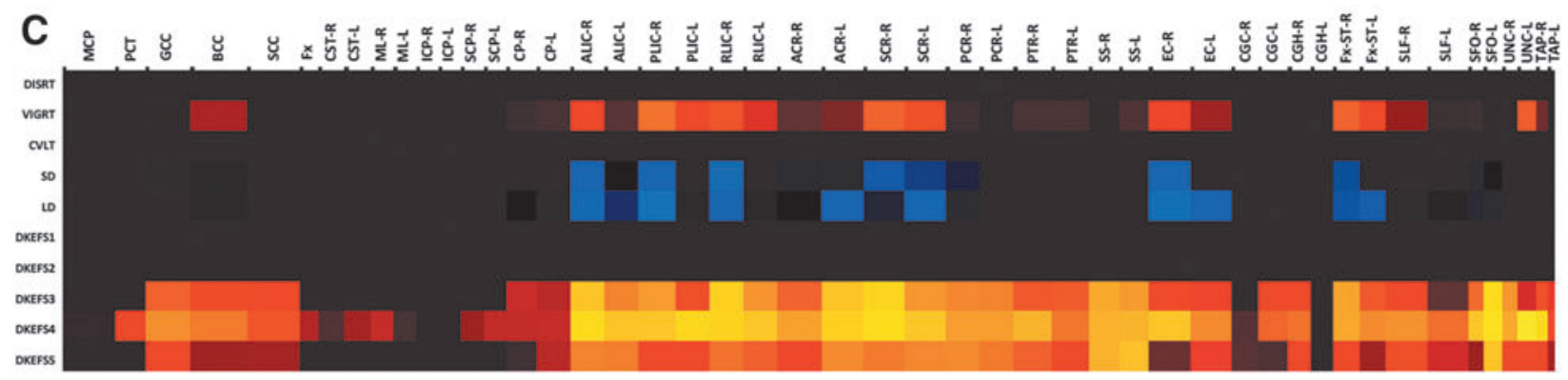

Negative correlation:

Positive correlation:

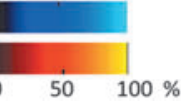




\section{Relationship of diffusion metrics to cognitive function: Within-group correlations}

Only $\mathrm{V}_{\mathrm{ic}}, \mathrm{P}_{0}$, FA, and RD had significant correlations with neuropsychological measures in mTBI subjects. An example of TBSS analysis on within-group correlations between diffusion metrics and neuropsychological measures is shown in Figure 4 for $V_{i c}$ and Number-Letter Switching (DKEFS4), a measure of executive function. Within the group of mTBI, $\mathrm{V}_{\text {ic }}$ and DKEFS4 had a wide spread of white matter voxels (approximately $5 \times 10^{4}$ ) that were positively correlated (corrected $p<0.05$ ). For all of the 70 diffusionneuropsychological pairs, the extent of the correlation expressed as the number of total significant voxels is summarized in Figure 5A.

$\mathrm{V}_{\text {ic }}$ correlated with 6 of 10 neuropsychological measures including vigilance attention (VIGRT), memory recall measures (SD and LD), and measures of executive function (DKEFS 3-5; Fig. 5A). As $\mathrm{V}_{\text {ic }}$ increased, performance decreased; that is, the number of words recalled on short and long delay (SD and LD) decreased, and increases were observed in the reaction time of vigilance tasks (VIGRT) and the time to complete in letter sequencing (DKEFS3), number letter switching (DKEFS4), and motor speed tasks (DKEFS5). Extent of the correlation ranged from approximately 2 to $5 \times 10^{4}$ voxels for the positive correlations (warm colors in Fig. 5A) and slightly lower, 0.5 to $1 \times 10^{4}$ voxels, for the negative correlations (cool colors in Fig. 5A). The secondmost sensitive diffusion metric was $\mathrm{P}_{0}$, which correlated with the same neuropsychological outcomes, except DKEFS3, and showed the same trends (Fig. 5A). For all the six $V_{\text {ic }}$-neuropsychological pairs with significant findings in TBSS, the collective correlation coefficients were between 0.83 and 0.87 (Fig. 5B). $\mathrm{P}_{0}$ had similar regression results (not shown here).

Anatomical distributions of white matter having significant correlations in $\mathrm{V}_{\text {ic }}$-neuropsychological pairs are summarized in the colorcoded matrix shown in Figure $5 \mathrm{C}$. The horizontal axis of the matrix denotes the JHU white matter label with the length of the interval reflecting its three-dimensional size in the atlas, and the color denotes the percentage of voxels in the labeled white mater tract with significant correlations. For the correlations with vigilance attention (VIGRT) and sustained memory (SD and LD), significant white matter involved three distinct white matter systems: interhemispheric fibers, longitudinal pyramidal tracts, and the limbic system. The interhemispheric fibers focused on the body of the corpus callosum; the involved pyramidal tracts included the external capsules, internal capsules, and their cortical projecting fibers; and the involved limbic system structures included the stria terminalis and uncinate fasciculus. Compared to attention and memory, the correlation between $\mathrm{V}_{\mathrm{ic}}$ and the executive functions (mainly DKEFS 3-5) was wider spread in white matter (Fig. 5C, bottom rows), except for the midbrain.

\section{Relationship of diffusion metrics to cognitive function: Between-group interactions}

The interaction effect of mTBI on the correlations is summarized in Figure 6, where significant findings describe significant group differences in the correlations between the diffusion metrics and neuropsychological measures. Five $\mathrm{V}_{\mathrm{ic}}$-neuropsychological pairs had significant between-group interactions in their correlations (Fig. 6A, fifth column). As shown in Figure 6B, for the memory tests (CVLT, SD, and LD), the direction of the correlations changed from a positive correlation in the trauma controls to a negative correlation in persons with mTBI. Similar results were observed for the executive function tests (DKEFS3 and 4), but the direction of change reversed. Goodness of fit for the interaction linear model ranged from $70 \%$ to $76 \%$ as indicated by the coefficient of determination $\left(\mathrm{R}^{2}\right)$ with a significance of $p_{\text {interaction }}<0.05$ for the moderator term in the model. Anatomical distributions of significant between-group interactions for $\mathrm{V}_{\mathrm{ic}}$ are summarized in the color-coded matrix in Figure 6C. Similar to the within-group correlations, white matter tracts having significant interaction effects were found in the corpus callosum, internal capsule and corona radiata, and limbic system (stria terminalis and uncinate fasciculus).

\section{Discussion}

In this study, $\mathrm{HYDI}^{53}$ was used to extract seven diffusion metrics computed from the DTI model, NODDI model, and the q-space imaging analysis. NODDI-derived $\mathrm{V}_{\mathrm{ic}}$, a potential proxy for in vivo axonal density measurements, and the q-space tissue restriction $\left(\mathrm{P}_{0}\right)$ index were consistently the most sensitive diffusion metrics for the between-group comparisons (only $\mathrm{V}_{\mathrm{ic}}$ ), within-group correlations, and group interaction effects. Specifically, $V_{i c}$ was lower in the white matter of patients with mTBI. The decreased density of axons may be explained by previous observations of axonal beading followed by apoptosis in infant mice shortly after closed TBI. ${ }^{16}$ Interestingly, the decrease of $\mathrm{V}_{\text {ic }}$ shortly after mTBI was in contrast to a previous study of $\mathrm{V}_{\text {ic }}$ on long-term effects (9-120 months after last concussion) of sports-related concussion, where increased $V_{i c}$ was found in 31 concussed athletes from seven varsity sport teams. ${ }^{60}$ The differences suggest biomechanical forces and (injury-to-imaging) time may play an important role on the trajectory of brain recovery.

Within the mTBI group, $\mathrm{V}_{\text {ic }}$ and $\mathrm{P}_{0}$ were associated with a majority of the neuropsychological outcome measures (Fig. 5A), but not the other trauma controls (Supplementary Fig. S1). (see online supplementary material at http://www.liebertpub.com)

Consistent with previous animal studies, ${ }^{17,18}$ these results suggest that measures of axonal density are related to cognitive

FIG. 6. Results of between-group interaction effects suggesting mild TBI as a moderator in the relationships between the diffusion metrics and neuropsychological outcome measures. (A) The color-coded matrix summarizes the total number of white matter voxels having significant interaction effects in the TBSS analyses. The cold colors indicate the correlation coefficient $(\rho)$ is lower in the mild TBI group compared to the control group, whereas the warm colors indicate the opposite. (B) Results of post-hoc regression analyses for the five $\mathrm{V}_{\mathrm{ic}}$-neuropsychological pairs that had significant TBSS results in (A). Each black dot denotes 1 individual in the trauma control group, and the red triangle denotes an individual in the mild TBI group. The coefficient of determination for the interaction model $\left(\mathrm{R}^{2}\right)$ and the significance of the moderator variable $\left(p_{\text {int }}\right)$ in the interaction model are reported in the subpanels. The interaction model was a general linear model with an interaction between the group membership (as moderator variable) and diffusion metrics: neuropsychological_outcome $=\beta_{0}+\beta_{1} \cdot$ group $+\beta_{2}$. diffusion_metric $+\beta_{3} \cdot \operatorname{group} \cdot \operatorname{diffusion} \_$metric + covariates + error. $(\mathbf{C})$ Anatomical distributions of white matter voxels with significant between-group interactions in the TBSS analyses of $\mathrm{V}_{\mathrm{ic}}$-neuropsychological measures. The horizontal axis denotes the Johns Hopkins University (JHU) white matter labels in Figure 1 with the length of the interval reflecting its three-dimensional size (in cubic root) in the atlas. The color denotes the percentage (\%) of voxels in the labeled white mater tract with significant interaction effects. mTBI, mild traumatic brain injury; TBSS, tract-based spatial statistics. 
A

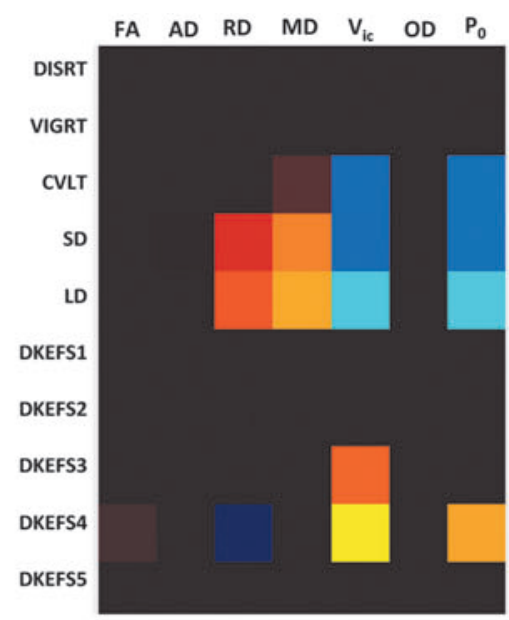

Negative $\left(\rho_{\text {mTBI }}-\rho_{\text {control }}\right)$ :
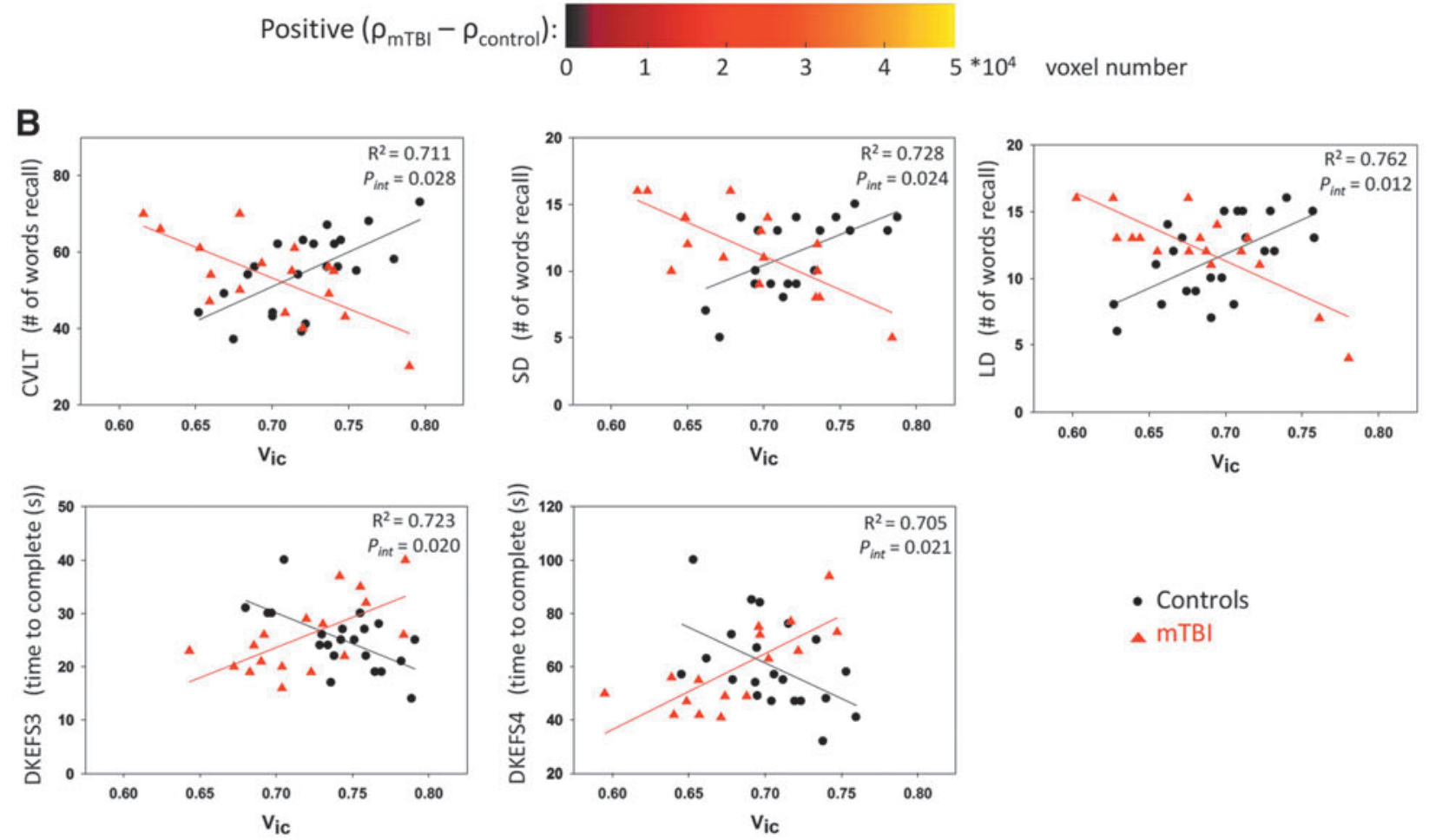

- Controls

a mTBI

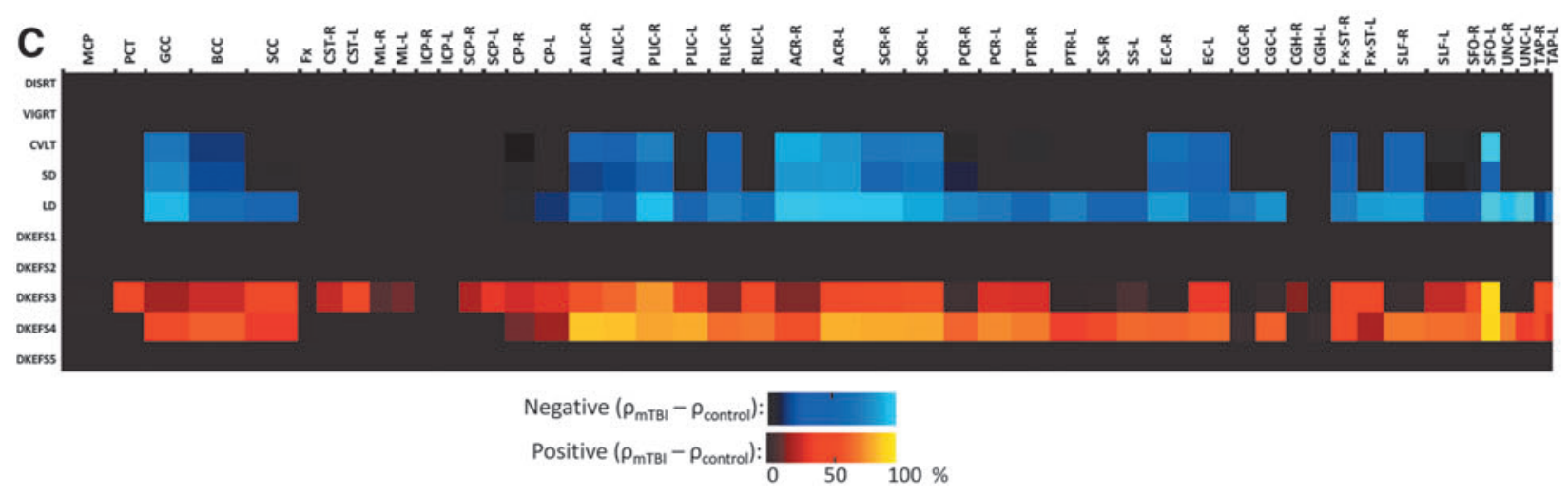


functioning. The relationship of axonal density to cognitive performance in mTBI patients (higher density associated with worse performance) was found to be opposite that observed in the other trauma control group, where higher axonal density was associated with better neuropsychological performance (Fig. 6B). A similar relationship was found with $\mathrm{P}_{0} . \mathrm{P}_{0}$ is the return to origin probability defined in the model-free q-space imaging theory ${ }^{89}$ and is often interpreted as a measure of restricted diffusion and cellularity. ${ }^{53,61,83}$ Under circumstances in which axons dominate the packing density, $\mathrm{P}_{0}$ may highly correlate with, and serve as, an indirect marker of axonal density. Similarly, we detected that individuals with lower tissue restriction in their white matter exhibited better cognitive performance post-mTBI. Potential underlying mechanisms for this finding may include a physical buffering effect inherent in the larger interstitial space attributed to lower axonal density or packing density. It is possible that larger interstitial spaces may cushion axons from biomechanical insults by absorbing and dissipating propagating energy. Alternatively, lower axonal density is likely to be associated with higher ratio of neurosupportive cells, such as astrocytes. Converging evidence suggests that astrocytes regulate and restrict inflammation, and help preserve brain function post-TBI. ${ }^{90}$ These observations of potential shielding/protecting effects of lower axonal density from TBIs call for further research, such as a carefully designed prospective study with MRI scans at baseline and after TBIs.

Spatial organization of axons remained stable, albeit with a decrease in density, post-mTBI. Axonal orientation dispersion (i.e., OD) in the NODDI model did not differ significantly between groups or correlate with any neuropsychological measures. This finding is somewhat supported by FA of the DTI model, in which significances were weak or nonexistent across the three-stage statistical tests. Both OD and FA represent the structural organization of white matter. The differences lie in that the DTI model does not account for differences between intra- and extra-axonal diffusion, whereas OD is modeled directly for axons. ${ }^{54}$ Nevertheless, previous studies using FA and cognitive functions reported similar negative TBSS or ROI results in 15 college athletes concussed within $30( \pm 2)$ days, ${ }^{91} 23$ emergency room patients injured within $17( \pm 7.2)$ days, ${ }^{92}$ and 61 level 1 trauma center patients injured within $47( \pm 6.5)$ days. ${ }^{93}$ However, it is possible that these studies, including ours, were underpowered to detect a small effect size of FA in $\mathrm{mTBI}$.

The aim of this study was to find common white matter regions that are susceptible to mTBI regardless of heterogeneity of the injury. Anatomical distribution of affected white matter focuses on major and long fiber tracts, including the corpus callosum, pyramidal tracts, and the anterior and superior corona radiata, consistent with other human TBI studies suggesting that these white matter regions are vulnerable to biomechanical injury. ${ }^{21,39,94}$ To control for injury heterogeneity arising from impact directionality and magnitude of the forces, one may consider individualized analyses by testing the diffusion maps of the injured individual against a template established from a group of control subjects. As a preliminary study to investigate the sensitivity of advanced diffusion metrics, multiple comparisons were not performed across the seven diffusion metrics and 10 neuropsychological measures attributed to potential dependence within the diffusion metrics and neuropsychological measures. Alternatively, a principle component analysis may be performed to reduce the dimensionality of tested variables before multiple comparison adjustments.
The results of this study provide a snapshot of white matter alterations shortly after mTBI, but are limited to a cross-sectional study design with a finite sample size. The white matter alterations were interpreted as differences between the mTBI and control groups rather than between the baseline (i.e., before brain injury) and after brain injuries, which could be better characterized by a prospective study design. In addition, a longitudinal study design with multiple follow-up time points could better describe the dynamic changes in white matter microstructures during brain recovery in the acute, subacute, and chronic phases post-mTBI.

Similar to all diffusion models, the NODDI model has limitations. A frequently discussed point is that NODDI uses rigid-stick geometry to represent healthy axons with a Watson distribution to describe their orientations. ${ }^{54}$ The rigid-stick model imposes strong constraints with fixed intra-axonal diffusivities and a tortuosity model for extra-axonal diffusion. ${ }^{95}$ Alternative options may be to model the axons using white matter track integrity based on diffusion kurtosis imaging ${ }^{96}$ with "flexible" sticks that yield estimates of intra-axonal diffusivities or to model the axons based on composite hindered and restricted model of diffusion ${ }^{97}$ without linking intra- and extra-axonal diffusivities. In these cases, however, different sets of diffusion constraints or many more diffusion encoding directions are required. Another consideration is that the suggested initial condition in the NODDI fitting (i.e., the intra-axonal longitudinal diffusivity) ${ }^{54}$ may not apply to pathological conditions; hence, it may over- or underestimate the underlying axonal density. ${ }^{98}$ As suggested in a previous work, ${ }^{98}$ one may perturb the initial conditions to optimize the results, which was not performed in this study because no differences in axial/longitudinal diffusivity in DTI were found between groups.

Despite the limitations, NODDI provides a clinically feasible approach with robust nonlinear data fitting to probe axonal density in vivo. NODDI findings have been correlated with a tau pathology model of Alzheimer's disease, ${ }^{99}$ applied in in vivo human studies of normal aging, ${ }^{55}$ classic galactosemia, ${ }^{100}$ neonatal encephalopathy, ${ }^{101}$ white matter development in the newborn, ${ }^{102}$ and $\mathrm{MS}^{103}$ and has yielded valuable and biologically specific microstructural information about neurological disorders of the human brain.

In summary, the NODDI-derived axonal density index and qspace measure for tissue restriction demonstrated superior sensitivity to white matter changes shortly after mTBI over classic DTI. Alterations of axonal density and tissue restriction correlated with cognitive function, and the direction of the correlations was moderated by mTBI. Further, long-range white matter tracts appeared to be more vulnerable.

\section{Acknowledgments}

The authors thank Mary L. Hynes and Marcia A. Davis for assistance with data collection. This study was supported by institutional grants from Indiana University-Purdue University Indianapolis ITDP to YCW and TWM, National Institutes of Health grant R21 NS075791 to YCW and TWM, R01 R01AG053993 to YCW, the NCAA-DoD Grand Alliance W81XWH-14-2-0151 to TWM, and a Project Development Team within the ICTSI NIH/ NCRR Grant Number UL1TR001108.

\section{Author Disclosure Statement}

No competing financial interests exist. 


\section{References}

1. Rutland-Brown, W., Langlois, J.A., Thomas, K.E., and Xi, Y.L. (2006). Incidence of traumatic brain injury in the United States, 2003. J. Head Trauma Rehabil. 21, 544-548.

2. Langlois, J.A., Rutland-Brown, W., and Wald, M.M. (2006). The epidemiology and impact of traumatic brain injury: a brief overview. J. Head Trauma Rehabil. 21, 375-378.

3. McAllister, T.W. (2008). Neurobehavioral sequelae of traumatic brain injury: evaluation and management. World Psychiatry 7, 3-10.

4. Zemek, R.L., Farion, K.J., Sampson, M., and McGahern, C. (2013). Prognosticators of persistent symptoms following pediatric concussion: a systematic review. JAMA Pediatr. 167, 259-265.

5. Smith, D.H., Johnson, V.E., and Stewart, W. (2013). Chronic neuropathologies of single and repetitive TBI: substrates of dementia? Nat. Rev. Neurol. 9, 211-221.

6. McAllister, T.W. (2011). Neurobiological consequences of traumatic brain injury. Dialogues Clin. Neurosci. 13, 287-300.

7. De Beaumont, L., Theoret, H., Mongeon, D., Messier, J., Leclerc, S., Tremblay, S., Ellemberg, D., and Lassonde, M. (2009). Brain function decline in healthy retired athletes who sustained their last sports concussion in early adulthood. Brain 132, 695-708.

8. McKee, A.C., Stern, R.A., Nowinski, C.J., Stein, T.D., Alvarez, V.E., Daneshvar, D.H., Lee, H.S., Wojtowicz, S.M., Hall, G., Baugh, C.M., Riley, D.O., Kubilus, C.A., Cormier, K.A., Jacobs, M.A., Martin, B.R., Abraham, C.R., Ikezu, T., Reichard, R.R., Wolozin, B.L., Budson, A.E., Goldstein, L.E., Kowall, N.W., and Cantu, R.C. (2013). The spectrum of disease in chronic traumatic encephalopathy. Brain 136, 43-64.

9. Levin, B., and Bhardwaj, A. (2014). Chronic traumatic encephalopathy: a critical appraisal. Neurocrit. Care 20, 334-344.

10. McAllister, T.W., Sparling, M.B., Flashman, L.A., and Saykin, A.J. (2001). Neuroimaging findings in mild traumatic brain injury. J. Clin. Exp. Neuropsychol. 23, 775-791.

11. Radiological Society of North America. (2017). RSNA Position Statement on Traumatic Brain Injury (TBI) Imaging. Radiological Society of North America: Oak Brook, IL.

12. Farkas, O., and Povlishock, J.T. (2007). Cellular and subcellular change evoked by diffuse traumatic brain injury: a complex web of change extending far beyond focal damage. Prog. Brain Res. 161, 43-59.

13. Buki, A., and Povlishock, J.T. (2006). All roads lead to disconnection?-traumatic axonal injury revisited. Acta Neurochir. (Wien) 148, 181-193; discussion, 193-184.

14. LaPlaca, M.C., Prado, G.R., Cullen, D., and Simon, C.M. (2009). Plasma membrane damage as a marker of neuronal injury. Conf. Proc. IEEE Eng. Med. Biol. Soc. 2009, 1113-1116.

15. Simon, C.M., Sharif, S., Tan, R.P., and LaPlaca, M.C. (2009). Spinal cord contusion causes acute plasma membrane damage. J. Neurotrauma 26, 563-574.

16. Dikranian, K., Cohen, R., Mac Donald, C., Pan, Y., Brakefield, D., Bayly, P., and Parsadanian, A. (2008). Mild traumatic brain injury to the infant mouse causes robust white matter axonal degeneration which precedes apoptotic death of cortical and thalamic neurons. Exp. Neurol. 211, 551-560.

17. Spain, A., Daumas, S., Lifshitz, J., Rhodes, J., Andrews, P.J., Horsburgh, K., and Fowler, J.H. (2010). Mild fluid percussion injury in mice produces evolving selective axonal pathology and cognitive deficits relevant to human brain injury. J. Neurotrauma 27, 1429-1438.

18. Henninger, N., Bouley, J., Sikoglu, E.M., An, J., Moore, C.M., King, J.A., Bowser, R., Freeman, M.R., and Brown, R.H., Jr. (2016). Attenuated traumatic axonal injury and improved functional outcome after traumatic brain injury in mice lacking Sarm1. Brain 139, 10941105.

19. Belanger, H.G., Vanderploeg, R.D., Curtiss, G., and Warden, D.L. (2007). Recent neuroimaging techniques in mild traumatic brain injury. J. Neuropsychiatry Clin. Neurosci. 19, 5-20.

20. Bigler, E.D., and Bazarian, J.J. (2010). Diffusion tensor imaging: a biomarker for mild traumatic brain injury? Neurology 74, 626-627.

21. Dodd, A.B., Epstein, K., Ling, J.M., and Mayer, A.R. (2014). Diffusion tensor imaging findings in semi-acute mild traumatic brain injury. J. Neurotrauma 31, 1235-1248.

22. McAllister, T.W., Ford, J.C., Ji, S., Beckwith, J.G., Flashman, L.A., Paulsen, K., and Greenwald, R.M. (2012). Maximum principal strain and strain rate associated with concussion diagnosis correlates with changes in corpus callosum white matter indices. Ann. Biomed. Eng. $40,127-140$.

23. Lipton, M.L., Kim, N., Park, Y.K., Hulkower, M.B., Gardin, T.M., Shifteh, K., Kim, M., Zimmerman, M.E., Lipton, R.B., and Branch, C.A. (2012). Robust detection of traumatic axonal injury in individual mild traumatic brain injury patients: Intersubject variation, change over time and bidirectional changes in anisotropy. Brain Imaging Behav. 6, 329-342.

24. Arfanakis, K., Haughton, V.M., Carew, J.D., Rogers, B.P., Dempsey, R.J., and Meyerand, M.E. (2002). Diffusion tensor MR imaging in diffuse axonal injury. AJNR Am. J. Neuroradiol. 23, 794-802.

25. Inglese, M., Makani, S., Johnson, G., Cohen, B.A., Silver, J.A., Gonen, O., and Grossman, R.I. (2005). Diffuse axonal injury in mild traumatic brain injury: a diffusion tensor imaging study. J. Neurosurg. 103, 298-303.

26. Miles, L., Grossman, R.I., Johnson, G., Babb, J.S., Diller, L., and Inglese, M. (2008). Short-term DTI predictors of cognitive dysfunction in mild traumatic brain injury. Brain Inj. 22, 115-122.

27. Lo, C., Shifteh, K., Gold, T., Bello, J.A., and Lipton, M.L. (2009). Diffusion tensor imaging abnormalities in patients with mild traumatic brain injury and neurocognitive impairment. J. Comput. Assist. Tomogr. 33, 293-297.

28. Kumar, R., Gupta, R.K., Husain, M., Chaudhry, C., Srivastava, A., Saksena, S., and Rathore, R.K. (2009). Comparative evaluation of corpus callosum dti metrics in acute mild and moderate traumatic brain injury: its correlation with neuropsychometric tests. Brain Inj $23,675-685$

29. Lipton, M.L., Gulko, E., Zimmerman, M.E., Friedman, B.W., Kim, M., Gellella, E., Gold, T., Shifteh, K., Ardekani, B.A., and Branch, C.A. (2009). Diffusion-tensor imaging implicates prefrontal axonal injury in executive function impairment following very mild traumatic brain injury. Radiology 252, 816-824.

30. Kinnunen, K.M., Greenwood, R., Powell, J.H., Leech, R., Hawkins, P.C., Bonnelle, V., Patel, M.C., Counsell, S.J., and Sharp, D.J. (2011). White matter damage and cognitive impairment after traumatic brain injury. Brain 134, 449-463.

31. Toth, A., Kovacs, N., Perlaki, G., Orsi, G., Aradi, M., Komaromy, H., Ezer, E., Bukovics, P., Farkas, O., Janszky, J., Doczi, T., Buki, A., and Schwarcz, A. (2013). Multi-modal magnetic resonance imaging in the acute and sub-acute phase of mild traumatic brain injury: can we see the difference? J. Neurotrauma 30, 2-10.

32. Bazarian, J.J., Zhong, J., Blyth, B., Zhu, T., Kavcic, V., and Peterson, D. (2007). Diffusion tensor imaging detects clinically important axonal damage after mild traumatic brain injury: a pilot study. J. Neurotrauma 24, 1447-1459.

33. Wilde, E.A., McCauley, S.R., Hunter, J.V., Bigler, E.D., Chu, Z., Wang, Z.J., Hanten, G.R., Troyanskaya, M., Yallampalli, R., Li, X., Chia, J., and Levin, H.S. (2008). Diffusion tensor imaging of acute mild traumatic brain injury in adolescents. Neurology 70 , 948-955.

34. Mayer, A.R., Ling, J., Mannell, M.V., Gasparovic, C., Phillips, J.P., Doezema, D., Reichard, R., and Yeo, R.A. (2010). A prospective diffusion tensor imaging study in mild traumatic brain injury. Neurology 74, 643-650.

35. Ling, J.M., Pena, A., Yeo, R.A., Merideth, F.L., Klimaj, S., Gasparovic, C., and Mayer, A.R. (2012). Biomarkers of increased diffusion anisotropy in semi-acute mild traumatic brain injury: a longitudinal perspective. Brain 135, 1281-1292.

36. Wilde, E.A., McCauley, S.R., Barnes, A., Wu, T.C., Chu, Z., Hunter, J.V., and Bigler, E.D. (2012). Serial measurement of memory and diffusion tensor imaging changes within the first week following uncomplicated mild traumatic brain injury. Brain Imaging Behav. 6, 319-328.

37. Mayer, A.R., Ling, J.M., Yang, Z., Pena, A., Yeo, R.A., and Klimaj, S. (2012). Diffusion abnormalities in pediatric mild traumatic brain injury. J. Neurosci. 32, 17961-17969.

38. Waljas, M., Lange, R.T., Hakulinen, U., Huhtala, H., Dastidar, P., Hartikainen, K., Ohman, J., and Iverson, G.L. (2014). Biopsychosocial outcome after uncomplicated mild traumatic brain injury. J. Neurotrauma 31, 108-124.

39. Eierud, C., Craddock, R.C., Fletcher, S., Aulakh, M., King-Casas, B., Kuehl, D., and LaConte, S.M. (2014). Neuroimaging after mild traumatic brain injury: review and meta-analysis. Neuroimage Clin. 4, 283-294. 
40. Jones, D.K. (2008). Studying connections in the living human brain with diffusion MRI. Cortex 44, 936-952.

41. Assaf, Y., and Pasternak, O. (2008). Diffusion tensor imaging (DTI)based white matter mapping in brain research: a review. J. Mol. Neurosci. 34, 51-61.

42. Pierpaoli, C., and Basser, P.J. (1996). Toward a quantitative assessment of diffusion anisotropy. Magn. Reson. Med. 36, 893-906.

43. Jones, D.K. (2004). The effect of gradient sampling schemes on measures derived from diffusion tensor MRI: a Monte Carlo study. Magn. Reson. Med. 51, 807-815.

44. Alexander, A.L., Hasan, K.M., Lazar, M., Tsuruda, J.S., and Parker, D.L. (2001). Analysis of partial volume effects in diffusion-tensor MRI. Magn. Reson. Med. 45, 770-780.

45. Alexander, D.C., Barker, G.J., and Arridge, S.R. (2002). Detection and modeling of non-gaussian apparent diffusion coefficient profiles in human brain data. Magn. Reson. Med. 48, 331-340.

46. Wu, Y.C. (2016). Diffusion MRI: Tensors and Beyond in Medical Physics. University of Wisconsin-Madison: Madison, WI, p. 150.

47. Wheeler-Kingshott, C.A., and Cercignani, M. (2009). About "axial" and "radial" diffusivities. Magn. Reson. Med. 61, 1255-1260.

48. Tournier, J.D., Mori, S., and Leemans, A. (2011). Diffusion tensor imaging and beyond. Magn. Reson. Med. 65, 1532-1556.

49. Pasternak, O., Sochen, N., Gur, Y., Intrator, N., and Assaf, Y. (2009). Free water elimination and mapping from diffusion MRI. Magn. Reson. Med. 62, 717-730.

50. Metzler-Baddeley, C., O'Sullivan, M.J., Bells, S., Pasternak, O., and Jones, D.K. (2012). How and how not to correct for CSFcontamination in diffusion MRI. Neuroimage 59, 1394-1403.

51. Chiang, C.W., Wang, Y., Sun, P., Lin, T.H., Trinkaus, K., Cross, A.H., and Song, S.K. (2014). Quantifying white matter tract diffusion parameters in the presence of increased extra-fiber cellularity and vasogenic edema. Neuroimage 101, 310-319.

52. Albi, A., Pasternak, O., Minati, L., Marizzoni, M., Bartrés-Faz, D., Bargalló, N., Bosch, B., Rossini, P.M., Marra, C., Müller, B., Fiedler, U., Wiltfang, J., Roccatagliata, L., Picco, A., Nobili, F.M., Blin, O., Sein, J., Ranjeva, J.P., Didic, M., Bombois, S., Lopes, R., Bordet, R., Gros-Dagnac, H., Payoux, P., Zoccatelli, G., Alessandrini, F., Beltramello, A., Ferretti, A., Caulo, M., Aiello, M., Cavaliere, C., Soricelli, A., Parnetti, L., Tarducci, R., Floridi, P., Tsolaki, M., Constantinidis, M., Drevelegas, A., Frisoni, G., and Jovicich, J.; PharmaCog Consortium. (2017). Free water elimination improves test-retest reproducibility of diffusion tensor imaging indices in the brain: a longitudinal multisite study of healthy elderly subjects. Hum. Brain Mapp. 38, 12-26.

53. Wu, Y.C., and Alexander, A.L. (2007). Hybrid diffusion imaging. Neuroimage 36, 617-629.

54. Zhang, H., Schneider, T., Wheeler-Kingshott, C.A., and Alexander, D.C. (2012). NODDI: practical in vivo neurite orientation dispersion and density imaging of the human brain. Neuroimage 61, 1000-1016.

55. Kodiweera, C., Alexander, A.L., Harezlak, J., McAllister, T.W., and Wu, Y.C. (2016). Age effects and sex differences in human brain white matter of young to middle-aged adults: a DTI, NODDI, and qspace study. Neuroimage 128, 180-192.

56. Kodiweera, C., and Wu, Y.C. (2016). Data of noddi diffusion metrics in the brain and computer simulation of hybrid diffusion imaging (HYDI) acquisition scheme. Data Brief 7, 1131-1138.

57. Wintermark, M., Sanelli, P.C., Anzai, Y., Tsiouris, A.J., and Whitlow, C.T.; American College of Radiology Head Injury Institute. (2015). Imaging evidence and recommendations for traumatic brain injury: advanced neuro- and neurovascular imaging techniques. AJNR Am. J. Neuroradiol. 36, E1-E11.

58. Sepehrband, F., Clark, K.A., Ullmann, J.F., Kurniawan, N.D., Leanage, G., Reutens, D.C., and Yang, Z. (2015). Brain tissue compartment density estimated using diffusion-weighted MRI yields tissue parameters consistent with histology. Hum. Brain Mapp. 36, 3687-3702.

59. Grussu, F., Schneider, T., Tur, C., Yates, R.L., Tachrount, M., Ianus, A., Yiannakas, M.C., Newcombe, J., Zhang, H., Alexander, D.C., DeLuca, G.C., and Gandini Wheeler-Kingshott, C.A.M. (2017). Neurite dispersion: a new marker of multiple sclerosis spinal cord pathology? Ann. Clin. Transl. Neurol. 4, 663-679.

60. Churchill, N.W., Caverzasi, E., Graham, S.J., Hutchison, M.G., and Schweizer, T.A. (2017). White matter microstructure in athletes with a history of concussion: comparing diffusion tensor imaging (DTI) and neurite orientation dispersion and density imaging (NODDI). Hum. Brain Mapp. 38, 4201-4211.

61. Ozarslan, E., Koay, C.G., Shepherd, T.M., Komlosh, M.E., Irfanoglu, M.O., Pierpaoli, C., and Basser, P.J. (2013). Mean apparent propagator (MAP) MRI: a novel diffusion imaging method for mapping tissue microstructure. Neuroimage 78, 16-32.

62. Descoteaux, M., Deriche, R., Le Bihan, D., Mangin, J.F., and Poupon, C. (2011). Multiple q-shell diffusion propagator imaging. Med. Image Anal. 15, 603-621.

63. Hosseinbor, A.P., Chung, M.K., Wu, Y.C., and Alexander, A.L. (2013). Bessel fourier orientation reconstruction (bfor): An analytical diffusion propagator reconstruction for hybrid diffusion imaging and computation of q-space indices. Neuroimage 64, 650-670.

64. Assaf, Y., Mayk, A., and Cohen, Y. (2000). Displacement imaging of spinal cord using q-space diffusion-weighted MRI. Magn. Reson. Med. 44, 713-722.

65. Wu, Y.C., Field, A.S., Duncan, I.D., Samsonov, A.A., Kondo, Y., Tudorascu, D., and Alexander, A.L. (2011). High b-value and diffusion tensor imaging in a canine model of dysmyelination and brain maturation. Neuroimage 58, 829-837.

66. Biton, I.E., Duncan, I.D., and Cohen, Y. (2006). High b-value qspace diffusion MRI in myelin-deficient rat spinal cords. Magn. Reson. Imaging 24, 161-166.

67. Assaf, Y., Ben-Bashat, D., Chapman, J., Peled, S., Biton, I.E., Kafri, M., Segev, Y., Hendler, T., Korczyn, A.D., Graif, M., and Cohen, Y. (2002). High b-value q-space analyzed diffusion-weighted MRI: application to multiple sclerosis. Magn. Reson. Med. 47, 115-126.

68. Assaf, Y., Chapman, J., Ben-Bashat, D., Hendler, T., Segev, Y., Korczyn, A.D., Graif, M., and Cohen, Y. (2005). White matter changes in multiple sclerosis: correlation of q-space diffusion MRI and 1h MRS. Magn. Reson. Imaging 23, 703-710.

69. Wu, Y.C., Field, A.S., and Alexander, A.L. (2008). Computation of diffusion function measures in q-space using magnetic resonance hybrid diffusion imaging. IEEE Trans. Med. Imaging 27, 858-865.

70. Wu, Y.C., Field, A.S., Whalen, P.J., and Alexander, A.L. (2011). Age- and gender-related changes in the normal human brain using hybrid diffusion imaging (HYDI). Neuroimage 54, 1840-1853.

71. Kay, T., Harrington, D.E., Adams, R., Anderson, T., Berrol, S., Cicerone, K., Dahlberg, C., Gerber, D., Goka, R., Harley, P., Hilt, J., Horn, L., Lehmkuhl, D., and Malec, J. (1993). Definition of mild traumatic brain injury. J. Head Trauma Rehabil. 8, 86-87.

72. First, M.B., Spitzer, R.L., Gibbon, M., and Williams, J.B.W. (1996). Structured Clinical Interview for DSM-IV Axis I Disorders, Clinician Version (SCID-CV). American Psychiatric Press: Washington, DC.

73. McAllister, T.W., Flashman, L.A., McDonald, B.C., and Saykin, A.J. (2006). Mechanisms of working memory dysfunction after mild and moderate TBI: evidence from functional MRI and neurogenetics. J. Neurotrauma 23, 1450-1467.

74. McAllister, T.W., Saykin, A.J., Flashman, L.A., Sparling, M.B., Johnson, S.C., Guerin, S.J., Mamourian, A.C., Weaver, J.B., and Yanofsky, N. (1999). Brain activation during working memory 1 month after mild traumatic brain injury: a functional MRI study. Neurology 53, 1300-1308.

75. Beck, A.T. (1987). Beck Depression Inventory Manual. The Psychological Corporation: San Antonio, TX.

76. Spielberger, C.D. (1983). State-Trait Anxiety Inventory. Consulting Psychologists Press: Palo Alto, CA.

77. Setsompop, K., Gagoski, B.A., Polimeni, J.R., Witzel, T., Wedeen, V.J., and Wald, L.L. (2012). Blipped-controlled aliasing in parallel imaging for simultaneous multislice echo planar imaging with reduced g-factor penalty. Magn. Reson. Med. 67, 1210-1224.

78. Wen, Q., Mustafi, S.M., Harezlak, J., Risacher, S.L., West, J.D., Tallman, E., Farlow, M.R., Unverzagt, F.W., Apostolova, L.G., Saykin, A.J., and Wu, Y.-C. (2017). Axonal density is associated with subjective cognitive decline (SCD) in older adults assessed using the Cognitive Change Index, in: submitted to Alzheimer's Association International Conference (AAIC) 2017, July 14-15, London, UK.

79. Manjon, J.V., Coupe, P., Concha, L., Buades, A., Collins, D.L., and Robles, M. (2013). Diffusion weighted image denoising using overcomplete local PCA. PLoS One 8, e73021.

80. Yamada, H., Abe, O., Shizukuishi, T., Kikuta, J., Shinozaki, T., Dezawa, K., Nagano, A., Matsuda, M., Haradome, H., and Imamura, Y. (2014). Efficacy of distortion correction on diffusion imaging: 
comparison of FSL eddy and eddy_correct using 30 and 60 directions diffusion encoding. PLoS One 9, e112411.

81. Basser, P.J., Mattiello, J., and LeBihan, D. (1994). MR diffusion tensor spectroscopy and imaging. Biophys. J. 66, 259-267.

82. Daducci, A., Canales-Rodriguez, E.J., Zhang, H., Dyrby, T.B., Alexander, D.C., and Thiran, J.P. (2015). Accelerated microstructure imaging via convex optimization (AMICO) from diffusion MRI data. Neuroimage 105, 32-44.

83. Avram, A.V., Sarlls, J.E., Barnett, A.S., Ozarslan, E., Thomas, C., Irfanoglu, M.O., Hutchinson, E., Pierpaoli, C., and Basser, P.J. (2016). Clinical feasibility of using mean apparent propagator (MAP) MRI to characterize brain tissue microstructure. Neuroimage $127,422-434$

84. Avants, B.B., Tustison, N.J., Song, G., Cook, P.A., Klein, A., and Gee, J.C. (2011). A reproducible evaluation of ANTs similarity metric performance in brain image registration. Neuroimage 54, 2033-2044.

85. Smith, S.M., Jenkinson, M., Johansen-Berg, H., Rueckert, D., Nichols, T.E., Mackay, C.E., Watkins, K.E., Ciccarelli, O., Cader, M.Z., Matthews, P.M., and Behrens, T.E. (2006). Tract-based spatial statistics: voxelwise analysis of multi-subject diffusion data. Neuroimage 31, 1487-1505.

86. Smith, S.M., and Nichols, T.E. (2009). Threshold-free cluster enhancement: addressing problems of smoothing, threshold dependence and localisation in cluster inference. Neuroimage 44, 83-98.

87. Nichols, T.E., and Holmes, A.P. (2002). Nonparametric permutation tests for functional neuroimaging: a primer with examples. Hum Brain Mapp 15, 1-25.

88. Oishi, K., Zilles, K., Amunts, K., Faria, A., Jiang, H., Li, X., Akhter, K., Hua, K., Woods, R., Toga, A.W., Pike, G.B., Rosa-Neto, P., Evans, A., Zhang, J., Huang, H., Miller, M.I., van Zijl, P.C., Mazziotta, J., and Mori, S. (2008). Human brain white matter atlas: identification and assignment of common anatomical structures in superficial white matter. Neuroimage 43, 447-457.

89. Callaghan, P.T. (1991). Principles of Nuclear Magnetic Resonance Microscopy. Clarendon: Oxford, UK

90. Burda, J.E., Bernstein, A.M., and Sofroniew, M.V. (2016). Astrocyte roles in traumatic brain injury. Exp. Neurol. 275, Pt. 3, 305-315.

91. Zhang, K., Johnson, B., Pennell, D., Ray, W., Sebastianelli, W., and Slobounov, S. (2010). Are functional deficits in concussed individuals consistent with white matter structural alterations: combined FMRI \& DTI study. Exp. Brain Res. 204, 57-70.

92. Messe, A., Caplain, S., Paradot, G., Garrigue, D., Mineo, J.F., Soto Ares, G., Ducreux, D., Vignaud, F., Rozec, G., Desal, H., PelegriniIssac, M., Montreuil, M., Benali, H., and Lehericy, S. (2011). Diffusion tensor imaging and white matter lesions at the subacute stage in mild traumatic brain injury with persistent neurobehavioral impairment. Hum. Brain Mapp. 32, 999-1011.

93. Lange, R.T., Iverson, G.L., Brubacher, J.R., Madler, B., and Heran M.K. (2012). Diffusion tensor imaging findings are not strongly associated with postconcussional disorder 2 months following mild traumatic brain injury. J. Head Trauma Rehabil. 27, 188-198.
94. Bigler, E.D. (2013). Neuroimaging biomarkers in mild traumatic brain injury (mTBI). Neuropsychol. Rev. 23, 169-209.

95. Stanisz, G.J., Szafer, A., Wright, G.A., and Henkelman, R.M. (1997). An analytical model of restricted diffusion in bovine optic nerve. Magn. Reson. Med. 37, 103-111.

96. Fieremans, E., Jensen, J.H., and Helpern, J.A. (2011). White matter characterization with diffusional kurtosis imaging. Neuroimage 58, $177-188$.

97. Assaf, Y., and Basser, P.J. (2005). Composite hindered and restricted model of diffusion (CHARMED) MR imaging of the human brain Neuroimage 27, 48-58.

98. Guerrero, J.M., Adluru, N., Kecskemeti, S.R., Davidson, R.J., and Alexander, A.L. (2016). Investigating the effects of intrinsic diffusivity on neurite orientation dispersion and density imaging (NODDI), in: ISMRM 24th Annual Meeting \& Exhibition 2016, May 7-13, Singapore.

99. Colgan, N., Siow, B., O'Callaghan, J.M., Harrison, I.F., Wells, J.A., Holmes, H.E., Ismail, O., Richardson, S., Alexander, D.C., Collins, E.C., Fisher, E.M., Johnson, R., Schwarz, A.J., Ahmed, Z., O’Neill, M.J., Murray, T.K., Zhang, H., and Lythgoe, M.F. (2015). Application of neurite orientation dispersion and density imaging (NODDI) to a tau pathology model of Alzheimer's disease. Neuroimage 125, 739-744.

100. Timmers, I., Zhang, H., Bastiani, M., Jansma, B.M., Roebroeck, A., and Rubio-Gozalbo, M.E. (2015). White matter microstructure pathology in classic galactosemia revealed by neurite orientation dispersion and density imaging. J. Inherit. Metab. Dis. 38, 295-304.

101. Lally, P., Zhang, H., Pauliah, S., Price, D., Bainbridge, A., Balraj, G., Cady, E., Shankaran, S., and Thayyil, S. (2014). 8.9 microstructural changes in neonatal encephalopathy revealed with the neurite orientation dispersion and density imaging (NODDI) model. Arch. Dis. Child Fetal Neonatal 99, Suppl. 1, A14.

102. Kunz, N., Zhang, H., Vasung, L., O’Brien, K.R., Assaf, Y., Lazeyras, F., Alexander, D.C., and Huppi, P.S. (2014). Assessing white matter microstructure of the newborn with multi-shell diffusion MRI and biophysical compartment models. Neuroimage 96, 288-299.

103. Mustafi, S.M., Kodiweera, C., Ford, J.C., Randolph, J.S., Wishart, H., and Wu, Y.C. (2015). Neurite orientation dispersion and density imaging (NODDI) in multiple sclerosis, in: ISMRM 23rd Annual Meeting \& Exhibition 2015, May 30-June 5, Toronto, Ontario, Canada.

Address correspondence to: $Y u$-Chien $W u, M D, P h D, D A B M P$ Department of Radiology and Imaging Sciences Indiana University School of Medicine 355 West 16th Street

Suite 4100 Indianapolis, IN 46202

E-mail: yucwu@iu.edu 